Tropical Journal of Pharmaceutical Research July 2020; 19 (7): 1541-1550

ISSN: $1596-5996$ (print); 1596-9827 (electronic)

(C) Pharmacotherapy Group, Faculty of Pharmacy, University of Benin, Benin City, 300001 Nigeria.

Review Article

http://dx.doi.org/10.4314/tjpr.v19i7.29

\title{
An ethnopharmacological review of Hyptis suaveolens (L.) Poit
}

\author{
Rong Li, Gengqiu Tang, Xile Liu, Junni Li, Dong Wang, Shengguo Ji* \\ School of Chinese Traditional Medicine, Guangdong Pharmaceutical University, Guangdong 510006, PR China
}

*For correspondence: Email: shengguo ji@163.com; Tel: +86-18027342788

Sent for review: 11 November 2019

Revised accepted: 22 June 2020

\begin{abstract}
This review aimed to provide a comprehensive overview of ethnobotanical uses, chemical constituents, posology, and toxicology of Hyptis suaveolens, and to address the significant medicinal benefits in order to promote its application. An extensive and systematic review of the literature was undertaken and all relevant abstracts and full-text articles analyzed and included in the review. A wide range of traditional uses are cited in the literature, ranging from uses for malaria, constipation, stomach problems, renal inflammation to external uses in repelling insects and treating injuries such as lacerations and burnrelated damage to skin and tissues. To date, pharmacological studies have demonstrated the significant activities of this plant that support uses such as antimicrobial, antidiabetic, antiulcer, and antiinflammatory. Numerous important phytochemicals, including 6 triterpenes, 8 diterpenes and 1 flavonoid have been isolated, identified and reported. The extracts and phytochemicals isolated from the plants show considerable potential for medicinal exploitation and utilization, including antimitotic, antiproliferative, cytotoxic, antioxidant, anti-inflammatory, antibacterial, antifungal, antiviral, anti-secretory, hepatoprotective, insecticidal, and acaricidal activities. As a medicinal plant, $\mathrm{H}$. suaveolens is endowed with immense exploitation and utilization value and is widely used worldwide Therefore, further studies to fully elucidate its medicinal potential are warranted.
\end{abstract}

Keywords: Hyptis suaveolens (L.) Poit, Ulcer Antimicrobial Inflammation, Diterpenes, Traditional medicine, Ethnopharmacology, Lamiaceae

This is an Open Access article that uses a fund-ing model which does not charge readers or their institutions for access and distributed under the terms of the Creative Commons Attribution License (http://creativecommons.org/licenses/by/4.0) and the Budapest Open Access Initiative (http://www.budapestopenaccessinitiative.org/read), which permit unrestricted use, distribution, and reproduction in any medium, provided the original work is properly credited.

Tropical Journal of Pharmaceutical Research is indexed by Science Citation Index (SciSearch), Scopus, International Pharmaceutical Abstract, Chemical Abstracts, Embase, Index Copernicus, EBSCO, African Index Medicus, JournalSeek, Journal Citation Reports/Science Edition, Directory of Open Access Journals (DOAJ), African Journal Online, Bioline International, Open-J-Gate and Pharmacy Abstracts

\section{INTRODUCTION}

Species of the family Lamiaceae, which are largely herbaceous and of economic importance, are found mostly in tropical, subtropical, and temperate parts of the world. Hyptis suaveolens (L.) Poit is belonging to the genus Hyptis and family Lamiaceae that includes 775 species worldwide, which are primarily found in South America. Plants of the Hyptis genus have been highlighted for numerous medicinal properties such as tumorigenic, antifertility, antimicrobial, mycotoxic, and phytotoxic activities [1]. Among these plants, Hyptis suaveolens (L.) Poit is an important traditional medicinal plant that was originally native to tropical America and is currently considered as a weed worldwide.

H. suaveolens (Fig. 1) is a fast-growing perennial and aromatic herb that is $0.4-2 \mathrm{~m}$ high with a 
quadrate stem that bears hair. The leaves are either ovate or obovate, generally measuring 3-5 $\mathrm{cm}$ long and 2-4 cm wide with serrulate margins and a long stalk while its petioles are up to $3 \mathrm{~cm}$ long. The plant starts flowering early at an age of 2-3 months and produces copious blue flowers in small cymes along branches that end with reduced leaves. The flowers are pollinated by numerous pollinators leading to enormous seed production [2-3].

$H$. suaveolens is commonly distributed in dense clumps along roadsides, in over-grazed pastures, and around stockyards throughout the tropics and subtropics, but is normally restricted to places where the soils have been profoundly disturbed. The plant is characterized by a strong minty smell when crushed. Most parts of this plant are used in medicine formulations for treating numerous ailments such as respiratory and gastrointestinal infections, indigestion, colic, stomachache, colds, fever, burns, wounds, cramps and various skin complaints and it is used as an antirheumatic and antisuporific bath [4-6].

The plant has different names in various countries (Table 1, Table 2, and Table 3). For example, it is locally known as Bushmint, alfazema-brava, bamburral, or tapera velha in Brazil [7]; chichinguaste in El Salvador [8]; Chanor Picnut in Nicaragua [9]; Chia or Chan in Mexico [10]; Shan Xiang or maolaohu in China; gros, baumes, or hiptis à odeur in French; Indischer, Andorn, Wohlriechender Andorn, or Buschminze in German; bilati tulsi or ganga tulsi in Hindi; lampesan, jukut bau, or mangkamang in Indonesian; nioi-niga-kusa in Japanese; malbar, hutan, or pokok kemangi in Malaysian; chío gorda or chía grande in Mexico; amotan, suobkabayo, or loko-loko in Filipino; bamburral or mentrasto-grande in Portuguese; issop in Russian; oregano, or cimarron in Spannish; and kara or maeng lak kha in Thai [11].

$H$. suaveolens also has numerous synonyms, such as Gnoteris cordata Raf., Hyptis congesta Leonard., Hyptis graveolens Schrank, Marrubium indicum Blanco, Schaueria graveolens (Blume) Hassk. Or Schaueria suaveolens (L.) Hassk [12].Over the past years, few studies have focused on reviewing $H$. suaveolens in the literature and we did not find any comprehensive reviews of $H$. suaveolens, although its growing regions, phytochemistry, toxicity, and food and medicinal uses have been summarized to a certain extent $[2,3,10,12]$. To acquire provide additional knowledge on the considerable applicability and usefulness of this plant, here, we present a review of $H$. suaveolens that include its characteristics, uses, chemical constituents, posology, phytochemistry and toxicology to facilitate the development and utilization of $H$. suaveolens.

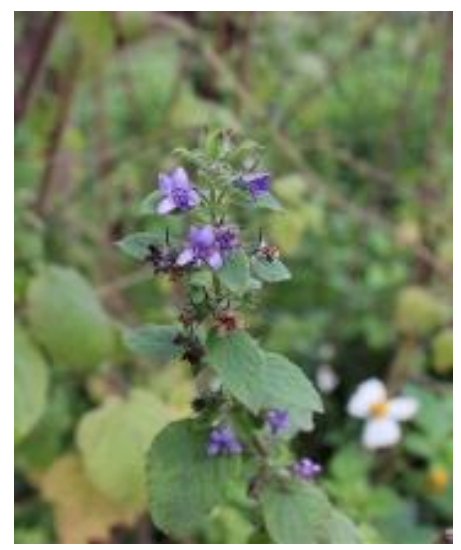

Figure 1: Hyptis suaveolens (L.) Poit (Lamiaceae)

\section{METHODS}

This review involved literature search on $H$. suaveolens (L.) Poit (Lamiaceae) using databases such as PubMed (http://www.ncbi. nlm.nih.gov/pubmed), ScienceDirect (https:// www.sciencedirect.com), and Web of Science (http://apps.webofknowledge.com) as well as search engines such as Baidu Scholar (http://xueshu.baidu.com) and Google Scholar (http://scholar.google.com). In confirming relevant entries, we considered publications up to the end of March 2020. Searches undertaken were found to be written in English, Portuguese, or Chinese.

\section{FINDINGS}

\section{Weed characteristics}

$H$. suaveolens, considered to be a weed worldwide, is currently ranked according to the importance of its use in different areas (Table 4). For instance, it is considered a serious weed in Brazil, a principal weed in Peru and Tanzania, but is a common weed in Australia, India, Micronesia, Philippines, Taiwan, and Thailand. This species is found in Cambodia, Ceylon (Sri Lanka), Congo-Kinshasa, Colombia, Costa Rica, Benin, Fiji, Ghana, Indonesia, Jamaica, Lebanon, Malaysia, Mauritius, Melanesia, Mexico, Netherlands, New guinea, Nicaragua, Panama, South Africa, Senegal, the US, Venezuela, and Vietnam, where it is also considered a weed, but of unknown ranked status. In China, the species is known to exist among the flora of the country, 
but confirmatory evidence of its weed status is needed [13].

\section{Distribution}

$H$. suaveolens is found across more than 50 countries in over the seven continents (Figure 2), including South American areas such as Brazil, Polynesia, Ecuador, French Guiana, Peru, Colombia, and Venezuela; Central American regions such as Belize, El Salvador, the Caribbean, Guatemala, Costa Rica, and Nicaragua; Latin American countries such as Honduras; North American areas such as Puerto Rico, Jamaica, Mexico, Panama, Trinidad and Tobago, Curacao, and the US; African countries such as Tanzania, Congo, Benin, Ghana, South Africa, Mauritius, Senegal, Nigeria, Burkina Faso, Guinea-Bissau, and Kenya.

In addition, it is also found in Oceanian countries such as Australia and New Guinea; Asian countries such as India, Bangladesh, Cambodia, Thailand, Indonesia, Lebanon, Malaysia, Vietnam, and China; Western Pacific countries including Micronesia and the Philippines; areas around the Indian Ocean such as Ceylon; Pacific regions such as Melanesia and Fiji; and European areas including the Netherlands. The literature survey revealed that only 22 developing countries among those listed here recorded the medicinal uses of this plant. $H$. suaveolens is more commonly used as a folkloric herbal medicine in developing countries [2].

\section{Traditional medicinal uses}

The different traditional medicinal uses of various parts of $H$. suaveolens in over 23 countries are summarized in Table 1, Table 2 and Table 3, where they are arranged by frequency of the same use in different countries. Five of these countries are located in Central America, four in Asia (Bangladesh, China, India, and Thailand), four in Africa (Tanzania, Nigeria, Burkina Faso, and Kenya) two in the Caribbean (Jamaica and Curaçao), and one in South America (Brazil). A wide range of traditional medicinal uses of this plant are reported in the literature. These applications range from in vivo use for conditions affecting the respiratory system, gastrointestinal tract, and gynecological system to in vitro conditions affecting the skin [53]. This plant appears to be most commonly reported to be used in the treatment of fever and headache (reported in fourteen countries), as an insect repellent, for stomach disorders, skin conditions, injuries (five countries each), weakness (two countries), abnormal leucorrhea, renal disorders, dysentery, and malaria (three countries each). In addition, the plant is also less frequently reported to be used for male disorders and menorrhagia (two countries each).

\section{Posology}

For headaches and colds, a decoction is prepared from 6-12 g of $H$. suaveolens for oral administration with a decoction of the fresh plant used to wash the body. In Bangladesh, $2 \mathrm{~g}$ of the seeds of $H$. suaveolens are soaked in water with mishri (crystalline sugar) for a whole day and then consumed for treating underweight [26]. In Bidar District of Karnataka in India, a spoonful $(10 \mathrm{~g})$ of the seed extract is administered orally once a day for 3 days as a remedy for leucorrhoea and temporary male infertility. Documentation of traditional knowledge of medicinal plants used in Bidar District and Karnataka reports that in Seshachalam Biosphere Reserve Forest of Chittoor District and Andhra Pradesh India, 4-7 $\mathrm{g}$ of the leaf powder is rolled in beedi leaves, which is then smoked to relieve colds and nasal congestion [36]. With an iron-deficient diet and high incidences of bloodsucking helminths, people in Bastimentos and Panama struggle with iron deficiency anemia. Consequently, a black drink prepared from $H$. suaveolens is the ethnopharmacological treatment for these pathologies, including iron deficiency anemia that is widely used in Bastimentos and unique to this community [54].

\section{Phytochemistry}

Research on the phytochemistry of $H$. suaveolens are has revealed that extracts of its different plant contain alkaloids, flavonoids, terpenoids, and tannins [55,56]. The saponin content of the leaves and stems is $6.10 \% \pm$ $0.42 \%$ and $10.50 \% \pm 0.79 \%$, respectively, while saponins have not been found in the roots. The contents of alkaloids, flavonoids, and tannins in the leaves are $2.80 \pm 0.28,1.90 \pm 0.14$, and 5.50 $\pm 0.074 \%$, respectively. Compared to the stem, the contents of alkaloids, flavonoids, and tannins are $1.60 \pm 0.00,0.30 \pm 0.14$, and $0.23 \pm 0.07 \%$.

\section{Chemical constituents}

Over the past years, the chemical constituents of $H$. suaveolens have been investigated widely in different the countries, and numerous new compounds have been isolated from this plant and their structures identified. In this paper, the dominant compounds isolated from different parts of $H$. suaveolens collected from various regions are summarized in Table 5 and Table 6. The structures of these compounds are mostly terpenoids including sesquiterpenes, diterpenes, 
triterpenes, and $\beta$-sitosterol. Most studies of the chemical constituents reported that they were mainly accumulated in the essential oil of the plant, but little attention has been focused on other extracts of $H$. suaveolens.

\section{Toxicity}

Extracts of $H$. suaveolens have shown effective insecticidal activity because of their toxicity to plant pests. The explanation for this biomechanism may be that the volatile oil of the extracts, which are characterized by a strong odor, can reduce the appetite of insects for the plants. The toxic effects of $H$. suaveolens are summarized in Table 7.

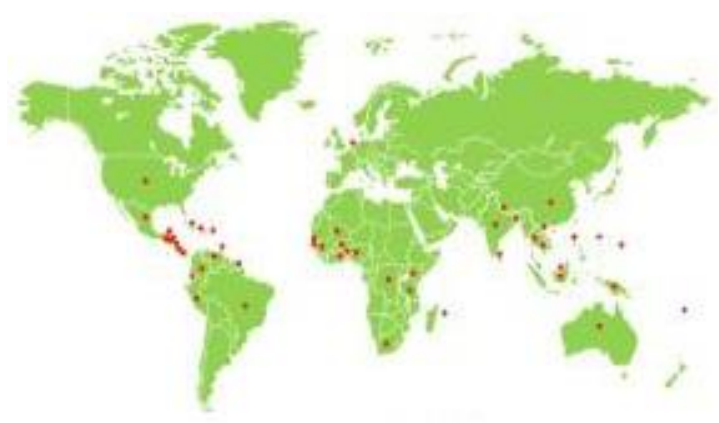

Figure 2: The distribution of Hyptis suaveolens in the world

Table 1: Ethnomedical uses of Hyptis suaveolens (L.) Poit (contd.)

\begin{tabular}{|c|c|c|c|c|}
\hline Country & Ethnomedical use & Plant part(s) & Preparation & Reference \\
\hline \multirow[t]{18}{*}{ Bangladesh } & Acidity, flatulence, gastric troubles & Seed & Sherbet $^{\mathrm{a}}\left(\right.$ int $\left.^{\mathrm{b}}\right)$ & 14 \\
\hline & Boils & Seed & $\begin{array}{l}\text { Maceration (applied aroun } \\
\text { boils) }\end{array}$ & \\
\hline & Boils & Root & Paste $\left(\operatorname{ext}^{c}\right)$ & 16 \\
\hline & Cancer, constipation, liver diseases & Seed, leaf & Not stated & 17 \\
\hline & Constipation & Leaf, bark & Not stated & 18 \\
\hline & \multicolumn{2}{|c|}{$\begin{array}{l}\text { Cooling agent, kidney disease, urinary tractSeed } \\
\text { dysuria, infections, laxative. }\end{array}$} & Sherbet ${ }^{a}\left(\right.$ int $\left.^{b}\right)$ & 19 \\
\hline & Gonorrhea & Seed & Along with other herbs & 20 \\
\hline & Headache & Whole plant & \multicolumn{2}{|c|}{$\begin{array}{l}\text { Crush (applied topically to the } 21 \\
\text { forehead) }\end{array}$} \\
\hline & Insect repellent & Whole plant & \multicolumn{2}{|c|}{$\begin{array}{l}\text { Dried and powdered whole plant } 21 \\
\text { is spread }\end{array}$} \\
\hline & Itching & Root & Extract & 16 \\
\hline & $\begin{array}{l}\text { Leucorrhea in women, low sperm densit) } \\
\text { in men. }\end{array}$ & yeed & Sherbet $^{\mathrm{a}}\left(\right.$ int $\left.^{\mathrm{b}}\right)$ & 22 \\
\hline & Loss of libido, to keep body cool & Seed & Sherbet $^{\mathrm{a}}\left(\right.$ int $\left.^{\mathrm{b}}\right)$ & 15 \\
\hline & Malaria & Whole plant & Crush $\left(\right.$ int $\left.^{\mathrm{b}}\right)$ & 21 \\
\hline & Physical weakness, sense of hotness in head & Seed & \multicolumn{2}{|c|}{$\begin{array}{l}\text { Powdered seeds are mixed with } 23 \\
\text { sugar }\end{array}$} \\
\hline & Stomach ache in children & Stem & \multicolumn{2}{|c|}{$\begin{array}{l}\text { Juice obtained from crushed } 24 \\
\text { stems is mixed with sugar }\end{array}$} \\
\hline & Stomach problems & Seed & Raw (int $\left.{ }^{b}\right)$ & 25 \\
\hline & To clear objects from eyes & Seed & Application of fruit to eyes & 25 \\
\hline & Underweight & Seed & Sherbet $^{\mathrm{a}}\left(\right.$ int $\left.^{\mathrm{b}}\right)$ & 26 \\
\hline \multirow[t]{3}{*}{ Brazil } & Diarrhea, digestive system, headache & Whole plant & Tea, syrup and infusion & 27 \\
\hline & Inflammation of the uterus and ovaries & Bark & Tea & 28 \\
\hline & Inflammatory, ulcer & Whole plant & Tea, bath & 29 \\
\hline \multirow[t]{2}{*}{ Burkina Faso } & Cold, cough & Leaf & Not stated & 30 \\
\hline & Insect repellent, itchy skin & Leaf, stem & Not stated & 31 \\
\hline \multirow[t]{9}{*}{ China } & Athlete's foot & Leaf & Pound $\left(\mathrm{ext}^{\mathrm{c}}\right)$ & 32 \\
\hline & Cold & Whole plant & Decoction (int ${ }^{b}$ ) & 33 \\
\hline & Diabetes, diarrhoea & Seed & Boiled (int $\left.{ }^{b}\right)$ & 32 \\
\hline & Eczema, dermatitis & Whole plant & $\begin{array}{l}\text { Decoction (wash affecte } \\
\text { area) }\end{array}$ & $\mathrm{d} 32$ \\
\hline & Eliminate toxin in the body & $\begin{array}{l}\text { Leaf (fresh) } \\
\text { wholeplant }\end{array}$ & orDecoction & 33 \\
\hline & Infertility & Root & Stew with chicken $\left(\right.$ int $\left.^{\mathrm{b}}\right)$ & 33 \\
\hline & Lobar seeper, pleurisy & Whole plant & Stew with pig lung or lean (int $\left.{ }^{b}\right)$ & 33 \\
\hline & Lymphoma & Root (fresh) & \multicolumn{2}{|c|}{$\begin{array}{l}\text { Stew with green-shell duck32, } 33 \\
\left.\text { eggs (int }{ }^{b}\right)\end{array}$} \\
\hline & Snakebite & Leaf (fresh) & Pound $\left(e^{e x t}{ }^{c}\right)$ & 32,33 \\
\hline
\end{tabular}


Table 2: Ethnomedical uses of Hyptis suaveolens (L.) Poit (contd.)

\begin{tabular}{|c|c|c|c|c|}
\hline Country & Ethnomedical use & Plant part(s) & Preparation & Reference \\
\hline \multirow[t]{9}{*}{ India } & Blood purifier & Root & Not stated & 34 \\
\hline & Boil, cuts, wounds & Leaf & Not stated & 34 \\
\hline & Chest pains, cough, wound healing & Whole plant & Not stated & 35 \\
\hline & Cold, fever and nasal congestion & Leaf & Beedi (inhalation) & 36 \\
\hline & Cough & Leaf & Eaten raw & 37 \\
\hline & $\begin{array}{c}\text { Menorrhagia, leucorrhoea, temporary male } \\
\text { sterility }\end{array}$ & Seed & Extract (intb) & 38 \\
\hline & Skin disease & Leaf & Not stated & 35 \\
\hline & Smoothing agent & Seed & Sherbet ${ }^{\mathrm{a}}$ & 39 \\
\hline & Wounds & Leaf & Juice $\left(e x t^{c}\right)$ & 40 \\
\hline \multirow[t]{7}{*}{ Nigeria } & Boils & Leaf & Juice $\left(e^{c} t^{c}\right)$ & 41 \\
\hline & Control the vector, mosquito & Whole plant & Smoke (smoking) & 42 \\
\hline & $\begin{array}{l}\text { Facilitate, childbirth, repel malaria-causing } \\
\text { insects }\end{array}$ & Leaf (fresh) & Extract $\left(\right.$ int $\left.^{\mathrm{b}}\right)$ & 43 \\
\hline & Headache & Whole plant & $\begin{array}{l}\text { Tied around the head until the ache } \\
\text { stops }\end{array}$ & 42 \\
\hline & Headache, mosquito repellant & Leaf (fresh) & Juice & 44 \\
\hline & Malaria & Leaf & Not stated & 45 \\
\hline & Mosquito repellent & Whole plant (fresh) & Raw & 42 \\
\hline \multirow[t]{4}{*}{ Philippines } & A stimulant if employed in rheumatism & Root & Decoction & 46 \\
\hline & Antirheumatic and antisuporific & Leaf and top & Baths & 47 \\
\hline & Antispasmodic & Leaf and top & Not stated $\left(\right.$ int $\left.^{\mathrm{b}}\right)$ & 47 \\
\hline & Appetizer & Root & Decoction & 46 \\
\hline \multirow[t]{4}{*}{ Senegal } & Expectorant & $\begin{array}{l}\text { Stem with } \\
\text { flower and seed }\end{array}$ & Infusion (int ${ }^{\mathrm{b}}$ ) & 48 \\
\hline & Headache and cold & Flower & Introduced into the nostrils & 48 \\
\hline & Migraine & $\begin{array}{l}\text { Powdered } \\
\text { plant part }\end{array}$ & Not stated & 48 \\
\hline & Tonic & $\begin{array}{l}\text { Stem with flower and } \\
\text { seed }\end{array}$ & Decoction (int $\left.{ }^{b}\right)$ & 48 \\
\hline \multirow[t]{3}{*}{ Tanzania } & Abdominal pains and general body weakness & Leaf & Vapours from boiling leave(inhalation) & 49 \\
\hline & Epileptic cases, psychosomatic & Leaf, stem & $\begin{array}{c}\text { Ashes (the leaves stems are burned } \\
\text { and ashes applied over scarifications } \\
\text { on the body) }\end{array}$ & 49 \\
\hline & Leukorrhoea & Root & Decoction $\left(\right.$ int $\left.^{\mathrm{b}}\right)$ & 49 \\
\hline \multirow[t]{3}{*}{ Thailand } & Antidiarrhoeal & Seed & Not stated & \\
\hline & Constipation & Seed & Dessert $\left(\right.$ int $\left.^{\mathrm{b}}\right)$ & 50 \\
\hline & Fever, fatigue & Whole plant & Decoction & \\
\hline \multirow[t]{2}{*}{ Trinidad } & $\begin{array}{l}\text { Colds, constipation, fever, flu, malaria, fever, } \\
\text { menorrhagia, yellow fever }\end{array}$ & Leaf & Tea & 51 \\
\hline & Common cold \& cough & Leaf & Infusion or crush and inhaled & 52 \\
\hline
\end{tabular}

Table 3: Ethnomedical uses of Hyptis suaveolens (L.) Poit (contd.)

\begin{tabular}{lllll}
\hline Country & Ethnomedical use & Plant part(s) & Preparation & Reference \\
\hline Trinidad & Fever & Leaf & Infusion or crush and inhaled 52 \\
& Flu & Leaf & Bath & 51 \\
& Cooling/cleanser & Leaf & Infusion or crush and inhaled 52 \\
\hline
\end{tabular}

a sherbet $=$ Seeds are soaked in water in which mishri (crystalline sugar) has been dissolved and taken as a drink; ${ }^{\text {bint }}=$ internal use; ${ }^{c}$ ext $=$ external use

Table 4: Posology of Hyptis suaveolens (L.) Poit.

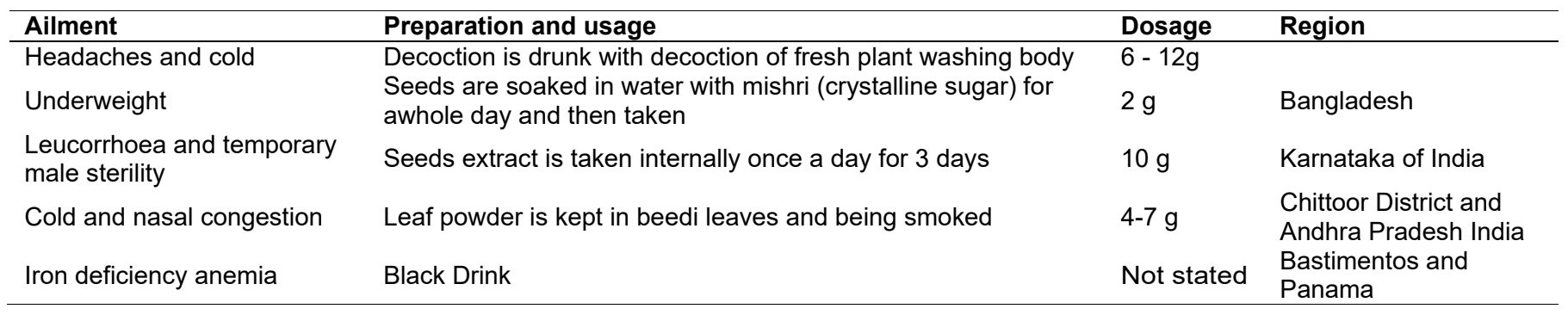


Table 5: Chemical constituents isolated from Hyptis suaveolens (L.) Poit (contd.)

\begin{tabular}{|c|c|c|c|}
\hline Dominant compounds & Isolated part & Plant source & Reference \\
\hline L-Fuco-4-O-methyl-D-glucurono-D-xylan & Seed-coat mucilage & Not stated & 57 \\
\hline $\begin{array}{l}\text { 1, 8-Cineole, -caryophyllene, -Copaene, -Phellandrene, } \\
\text {-elemene, eugenol }\end{array}$ & Essential oil & Darwin, Australia & 58 \\
\hline $\begin{array}{l}\text { sabinene, trans-a-bergamotene, } \beta \text {-caryophyllene, } \\
\text { terpinen- } 4 \text {-ol, } \beta \text {-pinene }\end{array}$ & Essential oil & Nigeria & 59 \\
\hline sabinene, $\beta$-caryophyllene, rrans-alpha-bergamotene & Essential oil & Mali & 60 \\
\hline $\begin{array}{l}\text { Sabinene, limonene, biclyclogermacrene, } \beta \text { - } \\
\text { phellandrene, } 1,8 \text {-cineole }\end{array}$ & Essential oil & Brazilian Cerrado & 61 \\
\hline Spathulenol, 1,8-cineole , ( E )-caryophyllene & Essential oil & Brazilian Cerrado & 62 \\
\hline $\begin{array}{l}\beta \text {-caryophyllene, } \beta \text {-elemene, } \\
\text { trans- } \alpha \text {-bergamotene, spathulenol, bicyclogermacrene }\end{array}$ & Essential oil & Tanzania & 63 \\
\hline $\begin{array}{l}\text { Sabinene, limonene, 1,8-cineole, ( E )-caryophyllene, } \\
\text { spathulenol }\end{array}$ & Essential oil & Brazil & 6 \\
\hline $\begin{array}{l}\alpha \text {-pinene, sabinene, } p \text {-cymene, terpinen-4-ol, } \\
\text { terpinolene, } 1,8 \text {-cineole, } \beta \text {-pinene, } \alpha \text {-terpinene, } \beta \text { - } \\
\text { caryophyllene, trans- } \alpha \text {-bergamotene }\end{array}$ & Essential oil & Nigeria & 64 \\
\hline $\begin{array}{l}\text { Fenchone-fenchol-chemotype, 1,8-cineole, } \\
\alpha \text {-terpinolene, sabinene }\end{array}$ & Essential oil & El Salvador & 65 \\
\hline sabinene, $\beta$-caryophyllene, bergamotol & Essential oil & Cameroon & 66 \\
\hline $\begin{array}{l}\text { abietane-type diterpenoid endoperoxide, } \\
13 \alpha \text { - epi -dioxiabiet-8(14)-en-18-ol }\end{array}$ & Leaves & southeastern Nigeria & 67 \\
\hline $\begin{array}{l}\text { terpenes spathulenol, globulol, dehydroabietol, a- } \\
\text { cadinol, beta-phellandrene }\end{array}$ & Essential oil & Brazil & 68 \\
\hline Sabinene, $\beta$-caryophyllene & Essential oil & Togo & 69 \\
\hline $\begin{array}{l}\text { 5-caranol, a-humulene, allo-aromadendrene, } \\
\text { Ermephilene, cis-sabinol, camphor }\end{array}$ & Essential oil & South India & 70 \\
\hline $\begin{array}{l}\text { sabinene, } 1,8 \text {-cineole, } \gamma \text {-terpinene, fenchone, fenchol, } \\
\text { the sesquiterpene } \beta \text {-caryophyllene }\end{array}$ & Essential oil & El Salvador & 71 \\
\hline $\begin{array}{l}\beta \text {-caryophyllene, } \alpha \text {-phellandrene, } \\
\text { caryophyllene oxide }\end{array}$ & Essential oil & Nigeria & 72 \\
\hline $\begin{array}{l}\beta \text {-caryophyllene, trans- } \alpha \text {-bergamotene, caryophyllene } \\
\text { oxide, } 6 \text {-hydroxycarvotanacetone, bicyclogermacrene, } \\
\text { (Z)-trans- } \alpha \text {-bergamotol, terpinen-4-ol. }\end{array}$ & Essential oil & Benin & 73 \\
\hline $\begin{array}{l}\text { Eucaliptoll, gama-ellemene, beta-pynene, } \\
(+) \text { - 3-carene, trans-beta-cariophyllene, germacrene }\end{array}$ & Essential oil & Brazil & 74 \\
\hline
\end{tabular}

Table 6: Chemical constituents isolated from Hyptis suaveolens (L.) Poit (contd.)

\begin{tabular}{|c|c|c|c|}
\hline Dominant compounds & Isolated part & Plant source & Reference \\
\hline $\begin{array}{l}\text { Monoterpene hydrocarbons, sesquiterpene } \\
\text { hydrocarbons, oxygenated monoterpenes, oxygenated } \\
\text { sesquiterpenes }\end{array}$ & Essential oil & Pisa, Italy & 75 \\
\hline $\begin{array}{l}\text { Sabinene, } \beta \text {-caryophyllene, terpinolene, } \beta \text {-pinene, } \\
\text { limonene, } 4 \text {-terpineol }\end{array}$ & Essential oil & Pisa, Italy & 76 \\
\hline $\begin{array}{l}\text { Isosuaveolic acid, 8,9-epoxysuaveolic acid, 14-O- } \\
\text { methylsuaveolic acid }\end{array}$ & Whole plant & $\begin{array}{l}\text { Nakhon Ratchasima, } \\
\text { Thailand }\end{array}$ & 77 \\
\hline $\begin{array}{l}\text { A-phellandrene, limonene, 1,8-cineole, fenchone, E- } \\
\text { caryophyllene, germacrene D }\end{array}$ & Leaves and flowers & Arauca, Colombia & 78 \\
\hline Suaveolic acid & Whole plant & Bangladesh & 79 \\
\hline 11S globulin (Hs11S) & Seed & Colima City, Mexico & 80 \\
\hline $\begin{array}{l}\text { 1,8-cineole, E-caryophyllene, sabinene, terpinolene, } \\
\text { bicyclogermacrene }\end{array}$ & Essential oil & Uttarakhand, India & 81 \\
\hline Galactoglucan, galactoglucomannan & Seed mucilage & North of Thailand & 82 \\
\hline $\begin{array}{l}\text { Caffeic acid, rutin, quercetin, } \\
\text { Citric acid, ferulic acid, gluconic acid } \\
\text { 3-O-beta-D-glucopyranoside, apigenin, sorbifolin, } \\
\text { quercetin, kaempferol, genkwanin, rosmarinic acid, } \\
\text { methyl rosmarinate, podophyllotoxin, } \\
\text { picropodophyllotoxin }\end{array}$ & Aqueous extract & Ghana & 84 \\
\hline
\end{tabular}


Table 7: Toxic effect of Hyptis suaveolens (L.) Poit.

\begin{tabular}{|c|c|c|c|c|}
\hline Toxic part & Toxicity & Value & Application & References \\
\hline Aqueous leaf extract & $\begin{array}{l}\text { Inhibit root growth of Allium } \\
\text { cepa }\end{array}$ & $\mathrm{EC}_{50}$ value of $1.92 \%$ & \multirow{4}{*}{$\begin{array}{l}\text { As herbal } \\
\text { insecticides/ } \\
\text { pesticides }\end{array}$} & 85 \\
\hline Ethanolic extract & $\begin{array}{l}\text { Toxic effect on larvae of } \\
\text { Aedesaegypti }\end{array}$ & $\begin{array}{l}\mathrm{LD}_{10} \text { value of } 0.01 \mathrm{ppm}, \mathrm{LD}_{50} \\
\text { value of } 0.60 \mathrm{ppm}, \mathrm{LD} 90 \text { value of } \\
1.45 \mathrm{ppm}\end{array}$ & & 86 \\
\hline Essential oil & $\begin{array}{l}\text { Toxic and repellent activity } \\
\text { against Sitophilus granarius } \\
\text { (L.) }\end{array}$ & $\begin{array}{l}\text { At the lowest dose }\left(2 \times 10^{-4} \quad \mu \mathrm{L} \text { oil }\right. \\
\left.\text { per } \mathrm{cm}^{2}\right)\end{array}$ & & 75 \\
\hline Essential oil & $\begin{array}{l}\text { Toxic effects on } \\
\text { Drosophilamelanogaster } \\
\text { and Artermiasalina }\end{array}$ & $\begin{array}{l}\mathrm{LC}_{50} \text { value of } 15.5 \text { and } 49.72 \\
\mu \mathrm{g} / \mathrm{mL} \text {, respectively }\end{array}$ & & 87 \\
\hline
\end{tabular}

\section{CONCLUDING REMARKS}

$H$. suaveolens is an important medicinal plant used in various indigenous herbal medicines for treating numerous diseases, as is clearly shown in the above-mentioned summary of its ethnobotanical, chemical, posology, and toxicological properties. Although there are numerous reports on the chemical composition and pharmacological properties of $H$. suaveolens, most studies were conducted on its essential oils. Therefore, more new compounds from other parts of this plant still need to be isolated and identified because the specific constituents mediating the pharmacological activities have not been identified. Safety assessments of $H$. suaveolens suggest that it has acute or chronic toxicity against grain pests, which suggests that it could be used as a herbal insecticide or pesticide. In addition, to further explore and exploit the therapeutic potential of $H$ suaveolens, quality control protocols are urgently needed to standardize this plant.

\section{DECLARATIONS}

\section{Acknowledgement}

The authors are very thankful for Guangdong Pharmaceutical University for providing the necessary facilities and encouragement to carry out this work successfully.

\section{Conflict of interest}

No conflict of interest is associated with this work.

\section{Contribution of authors}

We declare that this work was done by the authors named in this article and all liabilities pertaining to claims relating to the content of this article will be borne by the authors.

\section{Open Access}

This is an Open Access article that uses a funding model which does not charge readers or their institutions for access and distributed under the terms of the Creative Commons Attribution License (http://creativecommons.org/licenses/by/ 4.0) and the Budapest Open Access Initiative (http://www.budapestopenaccessinitiative.org/rea d), which permit unrestricted use, distribution, and reproduction in any medium, provided the original work is properly credited.

\section{REFERENCES}

1. Mcneil M, Facey $P$, Porter R. Essential oils from the Hyptis genus-a review (1909-2009). Nat Prod Commun 2011; 6: 1775-1796.

2. Sharma PP, Roy RK, Anurag, Dinesh G, Sharma VK. Hyptis suaveolens (L.) poit: A phyto-pharmacological review. Int J Chem Pharm Sci 2013; 4: 1-11.

3. Priya MD. A review on the pharmacology and phytochemistry of folklore medicinal plant Hyptis suaveolens (L.) Poit. Int. J. Basic, Appl. Innovative Res 2015; 4: 108 - 117.

4. Mahesh S, Chatterjee A, Pakrashi SC. The Treatise on Indian Medicinal Plants, Vol 5, (PID, New Delhi), 2001, 1.

5. Asekun OT, Ekundayo O, Adeniyi BA, Antimicrobial activity of the essential oil of Hyptis suaveolens leaves. Fitoterapia 1999; 70: 440-442.

6. Oliveira MJ, Campos IFP, Oliveira CBA, Santos MR, Souza PS, Santos SC, Seraphin JC, Ferri PH. Influence of growth phase on the essential oil composition of Hyptis suaveolens. Biochem System Ecol 2005; 33: 275-285.

7. Jesus $N$, Falcão $H$, Lima G, Caldas Filho M, Sales I, Gomes IF, Santos SG, Tavares JF, Barbosa-Filho JM, Batista LM. Hyptis suaveolens (L.) Poit (Lamiaceae), a medicinal plant protects the stomach against several gastric ulcer models. J Ethnopharmacol 2013; 150: 982988.

8. Grassi P, Reyes TSU, Sosa S, Tubaro A, Hofer O, ZitterlEglseer K. Anti-inflammatory activity of two diterpenes of 
Hyptis suaveolens from El Salvador. Z Naturforsch C 2006; 61: 165-170.

9. Barrett B, Kiefer D. Ethnomedical, biological and clinical support for medicinal plant use on Nicaragua's Atlantic coast, J Herbs Spices Med Plants 1997; 4: 77-108.

10. Solomon RA, Vergara SI. Hyptis suaveolens-Aprized weed. J. Nat. Con 1997; 9: 123-127.

11. Seidemann J. World spice plants, (Springer), 2005.

12. Ngozi LU, Ugochukwu N, Ifeoma PU, Charity EA, Chinyelu IE. The efficacy of hyptis suaveolens: a review of its nutritional and medicinal applications. Eur J Med Plants 2014; 4: 661-674.

13. Holm L, Pancho JV, Herberger JP, Plucknett DL. A geographical atlas of world weeds. Brittonia 1980; 32: 127-127.

14. Khan MA, Hasan MN, Jahan N, Das PR, Islam MT. Ethnomedicinal wisdom and famine food plants of the Hajong community of Baromari village in Netrakona district of Bangladesh. Am Eur J Sustain Agric 2012; 387-398.

15. ahmatullah M, Hasan ME, Islam MA, Islam MT, Jahan FI, Seraj S, Chowdhury AR, Jamal R, Islam MS, Miajee ZEU. A survey on medicinal plants used by the folk medicinal practitioners in three villages of Panchagarh and Thakurgaon district, Bangladesh. Am Eur J Sustain Agric 2010; 4: 291-301.

16. Rahman MA. Indigenous knowledge of herbal medicines in Bangladesh. 3. Treatement of skin diseases by tribal communities of the hill tracts districts. Bangladesh $\mathrm{J}$ Botany 2010; 39: 169-177.

17. Rahmatullah $M$, Mollik MAH, Khatun MA, Jahan $R$, Chowdhury AR. A survey on the use of medicinal plants by folk medicinal practitioners in five villages of Boalia sub-district, Rajshahi district, Bangladesh. Adv Nat Appl Sci 2010; 4: 39-44.

18. Rahmatullah M, Mollik AH, Paul AK, Jahan R, Khatun A, Seraj S, Chowdhury AR, Bashar A, Wahab R, Rahman T. A comparative analysis of medicinal plants used to treat gastrointestinal disorders in two sub-districts of Greater Khulna Division, Bangladesh Adv Nat Appl Sci 2010; 4: 22-29.

19. Hossan MS, Roy P, Seraj S, Mou SM, Monalisa MN, Jahan S, Khan S, Swarna A, Jahan R, Rahmatullah M. Ethnomedicinal knowledge among the Tongchongya tribal community of Roangchaari Upazila of Bandarban district, Bangladesh. Am Eur J Sustain Agric 2012; 6: 349-359.

20. Mia MM, Kadir MF, Hossan MS, Rahmatullah M. Medicinal plants of the Garo tribe inhabiting the Madhupur forest region of Bangladesh. Am Eur J Sustain Agric 2009; 3: 165-171.

21. Rahmatullah $M$, Mollik $A H$, Rahman $S$, Hasan $N$, Agarwala B, Jahan R. A medicinal plant study of the Santal tribe in Rangpur district, Bangladesh. J Altern Complem Med 2010; 16: 419-425.

22. Rahmatullah M, Das PR, Islam T, Ripa RJ, Hasan E. Medicinal plants and formulations of the Bongshi tribe of Bangladesh, Am Eur J Sustain Agric 2012; 6: 181-187.
23. Kabir MH, Hasan N, Rahman MM, Rahman MA, Khan $J A$, Hoque NT, Bhuiyan MR, Mou SM, Jahan $R$, Rahmatullah M. A survey of medicinal plants used by the Deb barma clan of the Tripura tribe of Moulvibazar district, Bangladesh. J Ethnobiol Ethnomed 2014; 10: 1.

24. Sarker MN, Mahin AA, Munira S, Akter S, Rahmatullah M. Ethnomedicinal plants of the Pankho community of Bilaichari Union in Rangamati district, Bangladesh. Am Eur J Sustain Agric 2013; 7: 114-120.

25. Shaheen EK, Syef A, Saha SS, Islam S, Al Hossain D. Medicinal plants used by the folk and tribal medicinal practitioners in two villages of Khakiachora and Khasia Palli in Sylhet district, Bangladesh. Adv. Nat. Appl. Sci 2011; 5: 100-111.

26. Rahmatullah $M$, Hasan A, Parvin W, Moniruzzaman $M$, Khatun A, Khatun Z, Jahan FI, Jahan R. Medicinal plants and formulations used by the Soren clan of the Santal tribe in Rajshahi district, Bangladesh for treatment of various ailments. Afr J Tradit Complement Altern Med 2012; 9: 350-359.

27. Gonçalves KG, Pasa MC. The ethnobotany and medicinal plants in Community Sucuri, Cuiabá, MT, Brazil, Interações (Campo Grande) 2015; 16: 245-256.

28. Pasa MC. Local knowledge and folk medicine: ethnobotany in Cuiabá, Mato Grosso, Brazil, Bol. Mus. Para. Emílio Goeldi. Cienc. Hum., Belém 2011; 6: 179 196.

29. Jesus NZTd, Lima JCdS, Silva RMd, Espinosa MM, Martins DTdO. Ethnobotanical survey of plants popularly used as anti-ulcer and anti-inflammatory in Pirizal, Nossa Senhora do Livramento, MT, Brazil. Rev Bras Farmacogn 2009; 19: 130-139.

30. Sourabié T, Some N, Bognonou O, Ouattara Y, Ouédraogo J. Ethnobotanical and ethnopharmacognostical survey on medicinal plants of Malon village and surrounding in the Cascades Region (Burkina Faso). losr J Pharm 2013; 3: 11-15.

31. Regina KMM, Adama H, Jeanne M, Odile N. Ethnobotany and Ethnopharmacognosy of Lamiaceae Species from Central Burkina Faso: Leucas martinicensis (Jacquin) $R$. Brown, Hoslundia opposita Vahl and Orthosiphon pallidus Royle Ex Benth. Am J Ethnomed 2015; 2: 219232.

32. Gang Rong L. Taiwan Qing Cao Yao Dui Zheng Tu Dian: Book one, (Northwest International Culture Co, Taiwan), 2009.

33. Society TCMP, Illustration of Medicinal Plants in Taitung, the second series, (WENHSIN PRESS, Taitung), 2012.

34. Kumar K, Abbas SG, Ethnomedicinal composition depends on floristic composition: A case studied in Sal forests of Jharkhand. Int J Pharm Life Sci 2012; 1710 1719.

35. Samydurai $P$, Jagatheshkumar $S$, Aravinthan $V$, Thangapandian $V$. Survey of wild aromatic ethnomedicinal plants of Velliangiri Hills in the Southern Western Ghats of Tamil Nadu, India. Int J Med Arom Plants 2012; 2: 229-234.

Trop J Pharm Res, July 2020; 19(7): 1548 
36. Ganesh P, Sudarsanam G. Ethnomedicinal Plants used by Yanadi Tribes in Seshachalam Biosphere Reserve Forest of Chittoor District, Andhra Pradesh India. Int J Pharm Life Sci 2013; 4.

37. Navaneethan P, Nautiyal S, Kalaivani T, Rajasekaran C. Cross-cultural ethnobotany and conservation of medicinal and aromatic plants in the Nilgiris, Western Ghats: A case study. Med Plants 2011; 3: 27-45.

38. Prashantkumar $P$, Vidyasagar G. Documentation of traditional knowledge on medicinal plants of Bidar district, Karnataka 2014.

39. Jain AK, Vairale MG, Singh R. Folklore claims on some medicinal plants used by Bheel tribe of Guna district Madhya Pradesh. Indian J Tradit Know 2010; 9: 105107.

40. Kumar A, Pandey VC, Singh AG, Tewari DD. Traditional uses of medicinal plants for dermatological healthcare management practices by the Tharu tribal community of Uttar Pradesh, India. Genet Resour Crop Ev 2013; 60: 203-224.

41. Bhat R, Etejere E, Oladipo V. Ethnobotanical studies from central Nigeria. Econ Bot 1990; 44: 382-390.

42. Nda-Umar UI, Gbate $M$, Umar AN, Mann $A$. Ethnobotanical study of medicinal plants used for the treatment of malaria in nupeland, north central Nigeria. Global J Res. Med. Plants \& Indigen. Med 2014; 3: 112.

43. Attah AF, O'Brien M, Koehbach J, Sonibare MA, Moody JO, Smith TJ, Gruber CW. Uterine contractility of plants used to facilitate childbirth in Nigerian ethnomedicine, J Ethnopharmacol 2012; 143: 377-382.

44. Ebenezer 10. Preliminary Investigations on the Ethnomedicinal Plants of Akoko Division, South West Nigeria. Glob. J. Hortic. Sci 2011; 3: 84-89.

45. Olorunnisola O, Adetutu A, Balogun E, Afolayan A. Ethnobotanical survey of medicinal plants used in the treatment of malarial in Ogbomoso, Southwest Nigeria. J Ethnopharmacol 2013; 150: 71-78.

46. Brown WH. Minor products of Philippine forests, Department of Agriculture and Natural Resources Bureau of Forestry. Manila 1921.

47. Stuartxchange. Philippine Medicinal Plants. 2014.

48. Kerharo J, Adam J. La pharmacopée sénégalaise traditionnelle, plantes médicinales et toxiques Edition Vigot frère. Paris, France, 1974.

49. Chhabra S, Mahunnah R, Mshiu E. Plants used in traditional medicine in Eastern Tanzania. III, Angiosperms (Euphorbiaceae to Menispermaceae). J Ethnopharmacol 1990; 28: 255-283.

50. Neamsuvan $O$, Singdam $P$, Yingcharoen $K$, Sengnon $N$. A survey of medicinal plants in mangrove and beach forests from sating Phra Peninsula, Songkhla Province, Thailand. J Med Plants Res 2012; 6: 2421-2437.

51. Wong W. Some folk medicinal plants from Trinidad. Econ Bot 1976; 30: 103-142.

52. Clement Y, Baksh-Comeau Y, Seaforth C. An ethnobotanical survey of medicinal plants in Trinidad. $J$ Ethnobiol Ethnomed 2015; 11: 1.
53. Picking D, Delgoda R, Boulogne I, Mitchell S. Hyptis verticillata jacq: a review of its traditional uses, phytochemistry, pharmacology and toxicology. J Ethnopharmacol 2013; 147(1): 16-41.

54. Nelsondooley $B$. The ethnomedical use of black drink to treat iron deficiency anemia in bastimentos, panama. Dooley 2004.

55. Prasanna S, Koppula S. Antimicrobial and preliminary phytochemical analysis of solvent extracts of Hyptis suaveolens from banks of River Krishna. Int. J. BioPharmacol. Res 2012; 1: 11-15.

56. Ijeh I, Edeoga $H$, Jimoh $M$, Ejeke C. Preliminary phytochemical, nutritional and toxicological studies of leaves and stems of Hyptis suaveolens. Res $J$ Pharmacol 2007; 1: 34-36.

57. Aspinall GO, Capek P, Carpenter RC, Gowda DC, Szafranek J. A novel l-fuco-4-o-methyl-d-glucurono-dxylan from hyptis suaveolens. Carbohydr Res 1991; 214: 107-113.

58. Peerzada. Chemical composition of the essential oil of hyptis suaveolens. Molecules 1997; 2: 165-168.

59. Asekun O, Olusegun Ekundayo. Essential oil constituents of Hyptis suaveolens (I.) poit. (bush tea) leaves from nigeria. J Essent Oil Res 2000; 12: 227-230.

60. Lassine Sidib Ã, Jean-Claude Chalchat, RaymondPhilippe Garry, Moussa Harama. Aromatic plants of mali (iii): chemical composition of essential oils of two Hyptis species: $h$. suaveolens (l.) poit. and h. spicigera lam. J Essent Oil Res 2001; 13: 55-57.

61. Azevedo NR, Campos IF, Ferreira HD, Portes TA, Santos SC, Seraphin JC, Paula JR, Ferri PH. Chemical variability in the essential oil of hyptis suaveolens. Phytochemistry 2001; 57: 733-776.

62. Azevedo NR, Campos IFP, Ferreira HD, Portes TA, Seraphin JC, Paula JRD, Santos SC, Ferri PH. Essential oil chemotypes in hyptis suaveolens, from brazilian cerrado. Biochem Syst Ecol 2002; 30: 205-216.

63. Malele RS, Mutayabarwa CK, Mwangi JW, Thoithi GN, Lopez AG. Essential oil of hyptis suaveolens (I.) poit. from Tanzania: composition and antifungal activity. J Essent Oil Res 2003; 15: 438-440.

64. Eshilokun AO, Kasali AA, Giwa-Ajeniya AO. Chemical composition of essential oils of two hyptis suaveolens, (I.) poit leaves from Nigeria. Flavour Frag J 2005; 20 : 528-530.

65. Grassi P, Nuñez MJ, Varmuza K, Franz C. Chemical polymorphism of essential oils of hyptis suaveolens from el salvador. Flavour Frag J 2005; 20: 131-135.

66. Tchoumbougnang F, Zollo PHA, Boyom FF, Nyegue MA, Bessière JM, Menut C. Aromatic plants of tropical central africa. xlviii. comparative study of the essential oils of four hyptis species from cameroon: $h$. lanceolata poit. h. pectinata (l.) poit. h. spicigera lam. and $h$. suaveolens poit. Flavour Frag J 2005; 20: 340-343.

67. Chukwujekwu JC, Smith P, Coombes PH, Mulholland $D A$, Van SJ. Antiplasmodial diterpenoid from the leaves of hyptis suaveolens. J Ethnopharmacol 2005; 102: 295297.

Trop J Pharm Res, July 2020; 19(7): 1549 
68. Martins FT, Santos MHD, Polo M, Barbosa LCDA. Chemical variation in the essential oil of hyptis suaveolens (I.) poit. under cultivation condition. Quím Nova 2006; 29: 1203-1209.

69. Koba K, Raynaud C, Millet J, Sanda JPCK. Chemical composition of hyptis pectinata I. h. lanceolata poit, $h$. suaveolens (I) poit and $h$. spicigera lam. essential oils from togo. J Essent Oil Bear PI 2007; 10: 357-364.

70. BeenaJoy, Omanakutty M, Mathew M. Antibacterial Effects and Chemical Composition of the Essential Oil of Hyptis suaveolens Poit Leaves. J Essent Oil Bear PI 2008; 11: 384-390.

71. Grassi P, Nuñez MJ, Reyes TSU, Franz C. Chemical variation in the essential oil composition of hyptis suaveolens (I.) poit. (lamiaceae), Nat Prod Commun 2008; 3: 1137-1140.

72. Akinolao O, Sundayo O, Guido F, Pierl C, Isiakaa O, Olayinky ET. Essential oil-bearing plants from Nigeria: studies on vernonia perrottettii (leaf and stem bark), young leaves from eucalyptus decaisneana and immature leaves of hyptis suaveolens. J Essent Oil Res 2009; 21: 154-158.

73. Kossouoh C, Moudachirou M, Adjakidje V, Chalchat JC, Figuérédo G. A comparative study of the chemical composition of the leaves and fruits deriving the essential oil of Hyptis suaveolens (I.) poit. from benin. $J$ Essent Oil Res 2010; 22: 507-509.

74. Moreira ACP, Lima EDO, Wanderley PA, Carmol ES, Souza ELD. Chemical composition and antifungal activity of Hyptis Suaveolens (L.) Poit leaves essential oil against Aspergillus species. Braz J Microbiol 2010; 41: 28.

75. Conti B, Canale A, Cioni PL, Flamini G, Rifici A. Hyptis suaveolens, and Hyptis spicigera, (lamiaceae) essential oils: qualitative analysis, contact toxicity and repellent activity against sitophilus granarius, (I.) (coleoptera: dryophthoridae). J Pest Sci 2011; 84: 219-228.

76. Benelli G, Flamini G, Canale A, Molfetta I, Cioni PL, Conti B. Repellence of hyptis suaveolens I. (lamiaceae) whole essential oil and major constituents against adults of the granary weevil sitophilus granarius (I.) (coleoptera: dryophthoridae). B Insectol 1967; 31: 177-183.

77. Prawatsri S, Suksamrarn A, Chindaduang A, Rukachaisirikul T. Abietane diterpenes from hyptis suaveolens. Chem Biodivers 2013; 10: 1494-1500.
78. Geovanna TG, Amner MA, Ana MC, Luisa FJ, Wilman $A D$. Componentes volátiles de Eriope crassipes, Hyptis conferta, $H$. dilatata, $H$. brachiata, $H$. suaveolens y $H$. mutabilis (Lamiaceae), Le problème de l'être chez Aristote: Presses. Universitaires de France 2014; 13: 727-730.

79. Islam AKMM, Ohno O, Suenaga K, Kato-Noguchi $H$. Suaveolic Acid: A Potent Phytotoxic Substance of Hyptis suaveolens. The Scientific World J 2014; 6.

80. Bojórquez-Velázquez E, Lino-López GJ, Huerta-Ocampo $J$ A, Barrera-Pachecoc A, Barba de la Rosa AP, Moreno A, Mancilla-Margalli NA, Osuna-Castro JA. Purification and biochemical characterization of $11 S$ globulin from chan (Hyptis suaveolens L. Poit) seeds. Food Chem 2016; 192: 203-211.

81. Kandpal V, Joshi PK, Joshi N. Chemical composition of leaf essential oil of Hyptis suaveolens (L.) Poit JICS 2017: 94: 201-203.

82. Praznik W, Čavarkapa A, Unger FM, Loeppert R, Holzer $W$, Viernstein $H$, Mueller M. Molecular dimensions and structural features of neutral polysaccharides from the seed mucilage of Hyptis suaveolens I. Food Chem 2017; 221: 1997-2004.

83. Ekow TN, Dzobo K, Adu F, Chirikure S, Wonkam A, Dandara C. Bush mint (hyptis suaveolens) and spreading hogweed (boerhavia diffusa) medicinal plant extracts differentially affect activities of cyp1a2, cyp2d6 and cyp3a4 enzymes. J Ethnopharmacol 2018; 211: 58.

84. Tang GQ, Liu XL, Gong X, Lin XJ, Lai XD, Wang D, Ji SG. Studies on the chemical compositions of Hyptis suaveolens (L.) Poit. J SERB CHEM SOC 2019; 84:245252.

85. Sumitha KV, Thoppil JE. Genotoxicity assessment of two common curing weeds: Hyptis suaveolens, (L.) Poir. and Leucas indica, (L.) R. Br. Cytotechnology 2016; 68: 115.

86. Amusan AA, Idowu AB, Arowolo FS. Comparative toxicity effect of bush tea leaves (Hyptis suaveolens) and orange peel (Citrus sinensis) oil extract on larvae of the yellow fever mosquito Aedes aegypti. Tanzan Health Res Bull 2005; 7: 174-178.

87. Bezerra JWA, Costa AR, Silva MAPD, Rocha MI, Boligon $A A$. Chemical composition and toxicological evaluation of Hyptis suaveolens (I.) poiteau (lamiaceae) in drosophila melanogaster and artemia salina. S Afr J Bot 2017; 113: 437-442. 